HNO 2008 $\cdot 56: 1229-1232$

DOI 10.1007/s00106-008-1675-4

Online publiziert: 15. März 2008

๑) Springer Medizin Verlag 2008

Redaktion

A. Berghaus, München
C. Storck ${ }^{1}$ S. Savic ${ }^{2} \cdot$ J. Egli ${ }^{1}$ C. Fischer ${ }^{1} \cdot$ M. Wolfensberger ${ }^{1}$

${ }^{1}$ Abteilung Phoniatrie, HNO-Klinik, Universitätsspital Basel, Schweiz

${ }^{2}$ Institut für Pathologie, Universitätsspital Basel, Schweiz

\title{
Granularzelltumor des Larynx
}

Granularzelltumoren (GZT) sind gutartige Neoplasien, ihr Ursprung ist neurogen [5]. Sie wurden erstmals 1926 von Abrikossoff beschrieben, weshalb sie gelegentlich auch als Abrikossoff-Tumoren bezeichnet werden [1]. Der Tumor tritt bevorzugt im Kopf-Hals-Bereich auf. Laryngeale GZT wurden aber lediglich in etwa 200 Fällen beschrieben [11]. Leitsymptom ist die Heiserkeit. Die meisten laryngealen GZT finden sich im dorsalen Drittel der Stimmlippe oder im Arytänoidbereich. Das klinische Bild lässt eine chronische Entzündung oder ein Malignom vermuten, sodass eine histologische Abklärung des Befunds unumgänglich ist. Wir stellen eine Patientin mit einem weiteren laryngealen GZT vor und diskutieren anhand des Falls die möglichen Therapieoptionen.

\section{Anamnese}

Die 57-jährige Frau stellte sich wegen einer seit 4 Monaten zunehmenden Heiserkeit vor. Anamnestisch bestand ein Nikotinabusus von über 6o Pack-Years. Atemnot, Dysphagie oder sonstige Beschwerden verneinte die Patientin.

\section{Befunde}

Lupenlaryngoskopisch sah man eine im dorsalen Drittel unregelmäßig verdickte linke Stimmlippe mit knotiger Veränderung der Mukosa im Bereich des Aryknorpels. Die vordere Kommissur war nicht einsehbar (• Abb. 1).

Zum Ausschluss eines Malignoms wurde in Intubationsnarkose eine Mikro- laryngoskopie mit Biopsie durchgeführt. Eine vollständige Exposition der Glottis war auch jetzt nicht möglich. Nur mittels $30^{\circ}$-Optik konnte die effektive Ausdehnung des Tumors bis zur vorderen Kommissur festgestellt werden (• Abb. 2). Die Läsion erschien sehr kompakt und derb, die Biopsie war nahezu unblutig.

Die histopathologische Untersuchung ergab die Diagnose eines GZT. In der Übersichtsvergrößerung sieht man eine pseudoepitheliomatöse Hyperplasie des Plattenepithels. Die Zellen des GZT sind rund und polygonal mit eosinophilem, PAS-positivem, fein granuliertem Zytoplasma und normalen Zellkernen (- Abb. 3, 4). Immunhistochemisch zeigt sich eine deutliche zytoplasmatische Positivität für den neurogenen Marker S-10o (- Abb. 5). Hinweise für Malignität wie Nekrosen, spindelige Zellen, Mitosen, Kernatypien oder Nukleolen fehlen.

\section{Therapie und Verlauf}

Da sich der Tumor über die ganze linke Stimmlippe bis zur vorderen Kommissur erstreckte und mikrolaryngoskopisch eine adäquate Exposition der Glottisebene nicht möglich war, wurde der Zugang über eine anteriore Thyreotomie gewählt. Unter operationsmikroskopischer Kontrolle wurde eine Chordektomie unter Mitnahme des Processus vocalis links durchgeführt. Damit war der Tumor im Bereich der Stimmlippe vollständig entfernt. Nach dorsal hin war eine komplette Tumorentfernung unter Einbezug des Arytänoidknorpels nicht möglich. Auf diese haben wir wegen der zu erwartenden postoperativen Aspirationsprobleme verzichtet. Eine Woche postoperativ bildete sich ei- ne tracheokutane Fistel, die jedoch innerhalb 10 Tagen spontan abheilte. Die initiale Aphonie besserte sich unter logopädischer Therapie.

Leider entzog sich die Patientin nach nur 9 Monaten der Kontrolle. Bis zu diesem Zeitpunkt ergaben sich keine Hinweise auf ein Tumorwachstum.

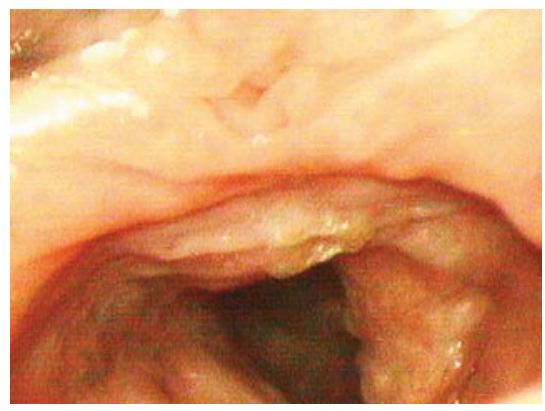

Abb. $1 \Delta$ Indirekt endoskopische Sicht auf den Granularzelltumor der linken Stimmlippe und posterioren Glottis

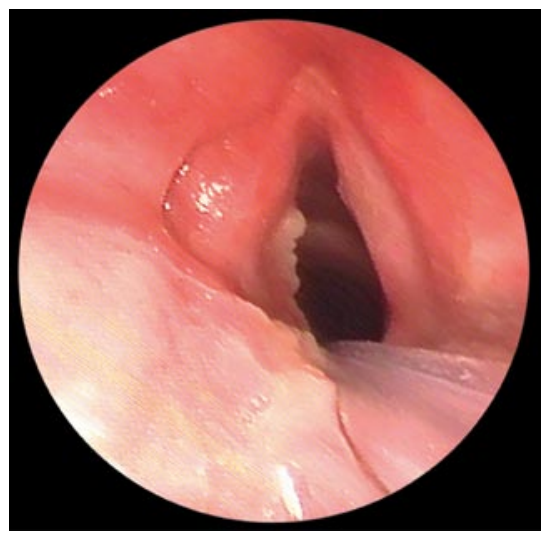

Abb. $2 \Delta$ Ausdehnung des Granularzelltumors in der linken Stimmlippe bis zur vorderen Kommissur 
HNO 2008 · 56:1229-1232

DOI 10.1007/s00106-008-1675-4

(c) Springer Medizin Verlag 2008

\section{Storck · S. Savic · J. Egli · C. Fischer · M. Wolfensberger \\ Granularzelltumor des Larynx}

\section{Zusammenfassung}

Granularzelltumoren (GZT) sind subkutan oder submukös gelegene, gutartige Tumoren neurogenen Ursprungs. Sie treten häufig im Kopf-Hals-Bereich auf, insbesondere in der Zunge. Bisher wurden etwa 200 laryngeale GZT beschrieben. Meist sind dabei die Stimmlippen und die posteriore Glottis betroffen. Eine Unterscheidung von einer chronischen Entzündung oder einem Malignom ist nur mittels Biopsie möglich. Als Therapie der Wahl wird die vollständige Exzision empfohlen, wobei Tumorausdehnung und zu erwartende Morbidität die Radikalität des Vorgehens bestimmen. Bei vollständiger Entfernung sind Rezidive selten.

\section{Schlüsselwörter}

Granularzelltumor · Larynx · Heiserkeit ·

Abrikossoff-Tumor · Zungentumor

\section{Granular cell tumor of the larynx: A case report}

\begin{abstract}
Granular cell tumors are benign subcutaneous or submucosal lesions of neurogenic origin. They are quite commonly found in the head and neck region, particularly in the tongue. However, only about 200 cases of laryngeal granular cell tumors have been reported so far. Most laryngeal granular cell tumors are located in the posterior part of the vocal fold and in the posterior commissure. Differentiation from inflammatory as well as from malignant lesions is possible only by biopsy. The treatment of choice is complete excision. However, tumor extension and expected morbidity of the surgery will inevitably influence the extent of the resection. Recurrences after complete excision are rare.
\end{abstract}

\section{Keywords}

Granular cell tumor - Larynx · Hoarseness . Abrikossoff tumor - Tumor of the tongue

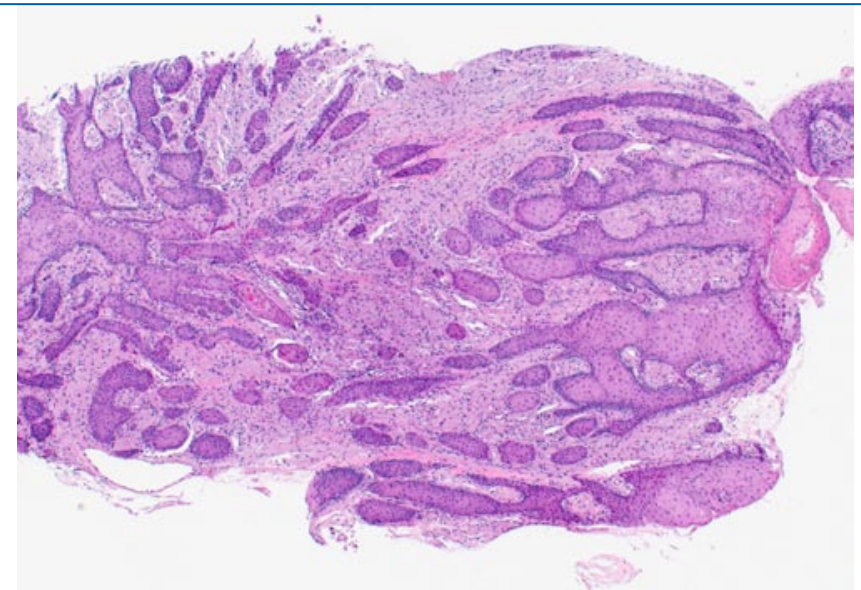

Abb. $3<$ Biopsie aus der linken Stimmlippe mit pseudoepitheliomatöser Hyperplasie des Plattenepithels (HE, Vergr. 100:1)

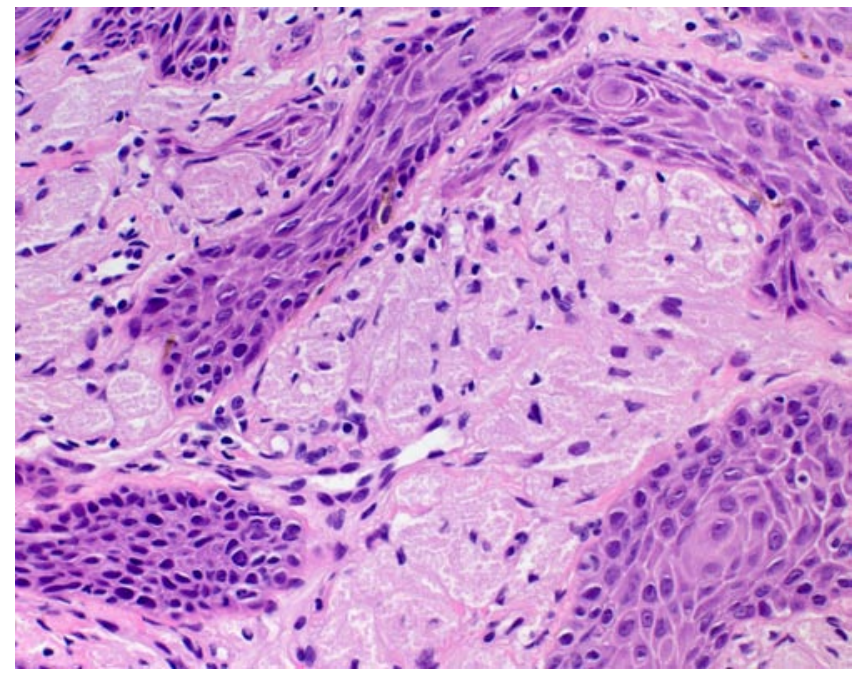

Abb. $4<$ Zwischen zytologisch blandem Plattenepithel der pseudoepitheliomatösen Hyperplasie liegen Zellen des Granularzelltumors (eosinophiles, fein granuliertes Zytoplasma mit normalen Zellkernen; HE, Vergr. 400:1)

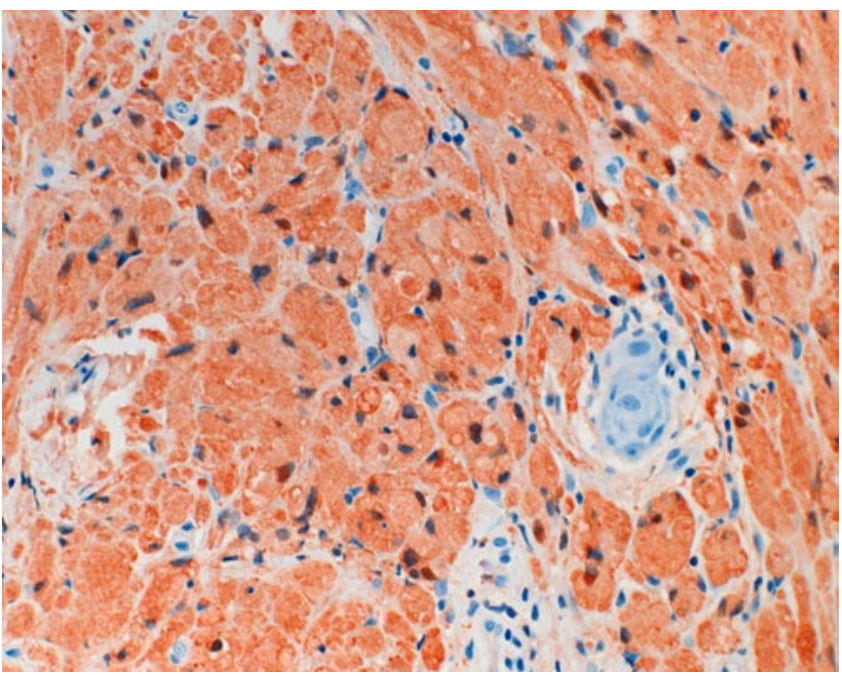

Abb. $5<$ Granularzelltumor mit starker Immunreaktivität für S100 (Vergr. 400:1)

\section{Diskussion}

Granularzelltumoren sind seltene, meist gutartige, vorwiegend subkutan oder submukös gelegene Tumoren. Beschrieben wurde der GZT erstmals 1926 von Abrikossoff, der einen myogenen Ursprung annahm und den Tumor darum als Myoblastom bezeichnete. Moderne immunhistochemische Untersuchungen haben dagegen bewiesen, dass der Tumor neurogenen Ursprungs ist.

$40 \%$ aller GZT finden sich im KopfHals-Bereich $[7,12]$, davon $75 \%$ in der 
Zunge [8]. Multifokale Manifestationen kommen in $15 \%$ aller Fälle vor [4]. Höchstens 1-2\% aller GZT sind maligne [4]. GZT finden sich vor allem bei Patienten im mittleren Lebensalter. Männer und Frauen sind ungefähr gleich häufig betroffen.

Eine laryngeale Manifestation ist ungewöhnlich. In der Literatur sind etwa 200 laryngeale GZT beschrieben, davon etwa 20 Fälle bei Kindern oder Jugendlichen [3, 10]. Leitsymptome sind Heiserkeit und seltener - Dysphagie. Meist ist das dorsale Drittel der Stimmlippe mit Übergang in die posteriore Glottis betroffen [10]. Einzig bei Patienten unter 17 Jahren wurden subglottische GZT beschrieben. Supraglottische Lokalisationen verursachen keine Symptome und werden meist nur zufällig entdeckt.

Klinisch ist eine Unterscheidung von einer entzündlichen Pathologie oder einem Malignom sehr schwierig, weshalb die Diagnose nur mittels Biopsie gestellt werden kann und dann meist den Operateur überrascht.

Die histologische Schnellschnittuntersuchung in unserem Fall weckte den Verdacht eines Granularzelltumors. Die histologisch häufig beobachtete pseudoepitheliomatöse Hyperplasie des Plattenepithels kann gelegentlich als invasives Plattenepithelkarzinom fehldiagnostiziert werden [6]. Diese Gefahr besteht vor allem bei kleinen und oberflächlichen Biopsien. Deshalb muss primär ausgiebig und tief biopsiert werden. Wir schlagen vor, trotz positivem Schnellschnittergebnis ein zweizeitiges Vorgehen zu wählen.

Therapie der Wahl ist die chirurgische Entfernung [2, 6, 7, 13]. Falls eine endoskopische Resektion nicht möglich ist, bietet sich ein externer Zugang an. Idealerweise sollte eine vollständige Entfernung angestrebt werden. Das Ausmaß der Resektion hängt aber von der Lage und der Ausdehnung des Tumors ab. $\mathrm{Da}$ das Ligamentum vocale meist mit reseziert werden muss, soll der Patient auf das Risiko einer bleibenden Heiserkeit aufmerksam gemacht werden. Eine ausgedehnte Exzision unter Mitnahme der Aryknorpels birgt zudem das Risiko einer oft nur schwer behandelbaren Dysphagie. Bei sehr ausgedehnten GZT mit Verlegung des Endolarynx wurden auch partielle und sogar totale Laryngektomien beschrieben [12].

In unserem Fall hätte eine vollständige Tumorentfernung eine Arytänoidektomie bedingt. In Übereinstimmung mit Autoren, die bei großen GZT von einer vollständigen Exzision abraten [9], haben wir wegen des postoperativen Aspirationsrisikos auf eine komplette Resektion verzichtet. Sollte es zu einem klinisch relevanten Rezidiv kommen, was gemäß Literatur in etwa $20 \%$ der Fälle zu erwarten ist [2], würden wir gegebenenfalls reintervenieren.

\section{Fazit für die Praxis}

Granularzelltumoren sind benigne, submukös gelegene Neoplasien neurogenen Ursprungs. Im Kopf-Hals-Bereich betreffen sie am häufigsten die Zunge. GZT des Kehlkopfs sind sehr selten. Neben den Stimmlippen befallen sie oft die hintere Kommissur. Sie können klinisch kaum von chronisch entzündlichen Prozessen und malignen Neoplasien unterschieden werden. Deshalb ist zur Diagnosesicherung immer eine Biopsie nötig. Therapie der Wahl ist eine vollständige Entfernung des GZT. In diesem Fall sind Rezidive selten. Ist eine vollständige Resektion mit inakzeptabler Morbidität verbunden, ist einem mehrzeitigen Vorgehen den Vorzug zu geben.

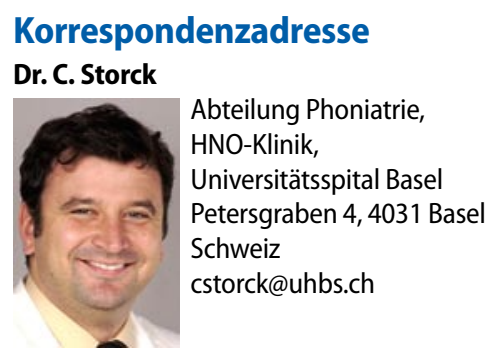

Interessenkonflikt. Keine Angaben.

\section{Literatur}

1. Abrikossoff A (1926) Über Myome, ausgehend von der quergestreiften willkürlichen Muskulatur. Virchows Arch Pathol Anat 260: 215-223

2. Alessi DM, Zimmermann MC (1988) Granular cell tumour of the head and neck. Laryngoscope 98: 810-814

\section{Hier steht eine Anzeige.}

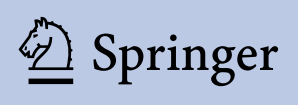


3. Conley S, Milbrath MM, Beste DJ (1992) Pediatric laryngeal granular cell tumor. J Otolaryngol 21 : 450-453

4. Curtis BV, Calcaterra TC, Coulson WF (1997) Multiple granular cell tumor: A case report and review of the literature. Head Neck 10: 634-637

5. Enzinger FM, Weiss SW (2001) Enzinger and Weiss's Soft tissue tumors. 4th edn. Mosby Inc, St. Louis, pp 1178-1187

6. Kamal SA, Othman EO (1998) Granular cell tumours of the larynx. J Larnygol Otol 112: 83-85

7. Krakamp B, Freudenhammer C, Köhler L et al. (2000) Granularzelltumor - eine seltene tumoröse Ösophagusläsion. Dtsch Med Wochenschr 125: 1511-1515

8. Lack EE, Worsham F, Callihan MD (1980) Granular cell tumor: A clinicopathological study of 110 patients. J Surg Oncol 12: 301-316

9. Morrison JG, Gray GF, Dao AH et al. (1987) Granular cell tumors. Am Surg 53: 156-160

10. Nolte E, Kleinsasser O (1982) Granularzelltumoren des Kehlkopfes. HNO 30: 333-339

11. Schottenfeld R, Marsh B (1997) Granular cell tumor of the larynx. Ear Nose Throat J 66: 56-59

12. Thawley SE, May M, Ogura JH (1974) Granular cell myoblastoma of the larynx. Laryngoscope 84: 1545-1551

13. Victoria LV, Hoffman HT, Robinson RA (1998) Granular cell tumour of the larynx. J Laryngol Otol 112: $373-376$

\section{G. G. Weismer ( (Hrsg.) Motor speech disorders}

\section{Essays for Ray Kent}

San Diego, Oxford, Brisbane: Plural Publishing, 2007, 315 S., (ISBN 1-59756-115-0), 79.95 USD

Motor speech disorders (MSD) sind Störungen der sensomotorischen Sprechplanung, des Programmierens der Kontrolle oder Exekution von Sprechbewegungen. Nicht zu den MSD gehören Defizite, die aufgrund struktureller Anomalien oder Defizite der Sprechwerkzeuge wie bei der Lippen-Kiefer-Gaumenspalte, der Laryngektomie, der Glossektomie, usw., entstehen. Zu MSD gehören weiterhin nicht die neurologischen Störungen, die durch höhere kognitive Probleme auftreten und die z.B. die linguistische Formulierung, den Inhalt oder die Form der gesprochenen Aussage betreffen. Somit können MSD unterteilt werden in die Dysarthrien und Sprechapraxien.

Zumindest bisher fallen unter den Begriff MSD auch nicht Störungen der sprechmotorischen Kontrolle wie z. B. das Stottern und die Aprosodie bei rechtshirnigen Dysfunktionen.

Das Buch von Gary Weismer widmet sich in neun Kapiteln dem MSD Ausführlich behandelt werden die neurologischen Grundlagen, die Sprechatmung, die segmentale Artikulation, die Rolle der Perzeption sowie die Dysphagie, die bei Patienten mit MSD ebenfalls auftreten kann Abgerundet wird das Buch mit einem Kapitel zu Interventionsmaßnahmen, um die Verständlichkeit und die Kommunikationsfähigkeit von Patienten mit Dysarthrie zu verbessern.

Die verschiedenen Kapitel sind von ehemaligen Studenten bzw. Mitarbeitern von Professor Ray Kent als Beitrag für eine Festschrift verfasst worden. Somit stellt das Buch kein klassisches Lehrbuch dar, es möchte vielmehr neben einem fundierten Überblick über das aktuelle wissenschaftliche Feld zu MSD auch das wissenschaftliche Lebenswerk von Kent würdigen.

Beide Ziele können als gut erreicht gelten. Hierzu trägt nicht nur die gelungene Themenauswahl, sondern auch die sorgfältige Aufarbeitung der aktuellen Literatur sowie der flüssige Schreibstil bei.

Das Buch kann sicherlich all denen empfohlen werden, die sich weiterführend mit
Dysarthrien und Apraxien bzw Sprechdyspraxien beschäftigen wollen. Möglicherweise werden jedoch diejenigen, die sich zum ersten Mal in die Materie einlesen wollen, ein klassisches Lehrbuch bevorzugen. Zumindest aber das 40 Seiten lange zweite Kapitel, das bereits einen guten Überblick über historische und aktuelle Aspekte zur Klassifikation, Symptomatologie, Diagnostik und Therapie der MSD gibt, kann nachhaltig zum Lesen empfohlen werden.

M. Ptok (Hannover) 